\title{
Prevalencia de alexitimia en los trastornos de la conducta alimentaria en una muestra clínica de $\mathbf{8 0 0}$ pacientes mexicanas
}

\author{
Prevalence of alexithymia in eating disorders in a clinical sample of 800 Mexican patients
}

\author{
Jorge Armando Barriguete-Meléndez ${ }^{1,2, *}$, Ana Pérez-Bustinzar ${ }^{1}$, Rocío Ivonne de la Vega-Morales ${ }^{1}$, \\ José Ángel Córdova-Villalobos², Jorge Manuel Sánchez-González², Paola Barriguete-Chávez Peón ${ }^{3}$ \\ y Luis Rojo-Moreno 4 \\ ${ }^{1}$ Clínicas Ángeles TCA; ${ }^{2}$ Academia Mexicana de Cirugía; ${ }^{3}$ Instituto Nacional de Perinatología. Ciudad de México, México; ${ }^{4}$ Universidad de Valencia, \\ Valencia, España
}

\begin{abstract}
Resumen
Introducción: La incapacidad para identificar y expresar sentimientos, y no distinguir entre las emociones y las sensaciones corporales, se conoce como alexitimia. En 1988 se desarrolló la Escala de Alexitimia de Toronto (TAS-20), que está conformada por 20 reactivos y tres factores: a) dificultad para identificar los sentimientos y las diferencias entre sentimientos y sensaciones corporales; b) dificultad para describir sentimientos; y c) pensamiento orientado externamente. Se considera que las personas con trastornos de la conducta alimentaria (TCA) tienen déficits específicos en la identificación y la comunicación de sus sentimientos. Objetivo: Realizar la validación de dicho instrumento. Método: Estudio de tipo transversal y de carácter psicométrico con diseño de una sola muestra, conformada por 435 personas con diagnóstico de TCA, con un rango de edad de 12 a 68 años, de las cuales el 91\% eran mujeres y el 9\% eran hombres. Para obtener la confiabilidad del instrumento se aplicó una prueba de consistencia interna, que dio como resultado un alfa de 0.89; posteriormente se hizo un análisis factorial de componentes principales con rotación oblimin. Resultados: De acuerdo con el análisis estadístico, se eliminaron seis reactivos, de tal forma que la escala finalizó con 14 reactivos, y al analizarlos se observó que concordaban con los dos factores principales de la escala original. Las pacientes con TCA presentan alexitimia. Discusión: La escala satisface los criterios de validez necesarios para ser utilizada en dicha población.
\end{abstract}

PALABRAS CLAVE: Alexitimia. Escala de Toronto. Validación. Trastornos de la conducta alimentaria. México.

\begin{abstract}
Introduction: The inability to identify, express feelings, and not distinguish between emotions and bodily sensations, is known as alexithymia. In 1988, it developed The Toronto Alexithymia Scale (TAS-20), consists of 20 items and three factors: a) difficulty of identifying feelings and differences between feelings and bodily sensations; b) difficulty of describing feelings; and c) externally oriented thinking. It's considered that people with eating disorders have specific deficits in identify and communicate their feelings. Objective: The present study has as purpose to the instrument validation. Methods: It was a cross-sectional study and psychometric character design of a single sample, formed of 435 persons suffering eating disorder (ED), with an age range of 12-68 years, of which $91 \%$ were women and $9 \%$ were men. To obtain the reliability of the instrument, applies internal consistency test, which resulted in an alpha of 0.89 , then applied a factor analysis of principals components with oblimin rotation. Results: According to statistical analysis, were eliminated six items, so the scale finished with 14 items, and
\end{abstract}

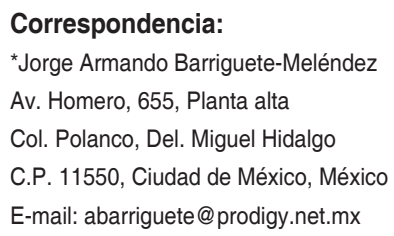

Fecha de recepción: 08-11-2016

Fecha de aceptación: 16-08-2017

DOI://dx.doi.org/10.24875/CIRU.M18000001
Cir Cir. 2018;86:43-49

Disponible en PubMed www.cirugiaycirujanos.com 
to analyze it observed that these items correspond with the two main factors of the original scale. The ED patients present alexithymia. Discussion: The scale satisfies the criteria of validity necessary for use in this population.

KEY WORDS: Alexithymia. Toronto Scale. Validation. Eating disorder. Mexico.

\section{Introducción}

Las emociones son experiencias internas que solo se manifiestan externamente mediante gestos o movimientos; nos inducen a actuar y por medio de ellas se provoca un determinado estado fisiológico en el cuerpo humano. Por su parte, los sentimientos son la expresión mental de las emociones. En contraste, existe un síndrome que lleva por nombre alexitimia ${ }^{1} y$ que se caracteriza por la incapacidad de identificar $y$ expresar sentimientos, además de no distinguir entre las emociones y las sensaciones corporales.

Ahora bien, la etimología de la palabra «alexitimia» proviene del griego: a (carencia), lexis (palabra) y thymos (emoción); se describe como un constructo multidimensional que significa la ausencia de palabras para expresar emociones ${ }^{2}$.

Entre las características que presentan las personas que padecen alexitimia se pueden mencionar las siguientes: a) dificultad marcada para identificar, describir y comunicar las emociones; b) dificultad para distinguir entre los sentimientos y las emociones, por un lado, y las sensaciones corporales, por el otro; c) deterioro de la capacidad de simbolización, que se manifiesta por una pobreza de fantasías y de otras actividades imaginativas; y d) preferencia para enfocar cognoscitivamente acontecimientos externos más que experiencias internas ${ }^{3}$.

Por otra parte, los trastornos de la conducta alimentaria (TCA) constituyen un grupo de trastornos mentales caracterizados por una conducta alterada ante la ingestión alimentaria o la aparición de comportamientos encaminados a controlar el peso. Esta alteración ocasiona problemas físicos y del funcionamiento psicosocial, y tiene como características principales un comportamiento distorsionado de la alimentación y una extrema preocupación por la imagen y el peso corporal ${ }^{4,5}$. López y Treasure $^{6}$ definen los TCA como enfermedades de salud mental complejas y multicausales, que afectan principalmente a adolescentes y mujeres jóvenes.

El diagnóstico más frecuente durante la adolescencia, de acuerdo con el Manual Diagnóstico y Estadístico de los Trastornos Mentales (DSM-TR, por sus siglas en inglés) de 2002, es el de TCA no especificado, seguido por el de anorexia nerviosa (AN) y el de bulimia nerviosa (BN). Sin embargo, los TCA no se han considerado un problema de salud pública en México, por lo que las estadísticas al respecto son escasas ${ }^{7}$.

La mayoría de los estudios sobre epidemiología de los TCA se basan en las conductas alimentarías de riesgo, las cuales se definen como aquellas conductas compensatorias inapropiadas características de los TCA que no cumplen los requerimientos diagnósticos tanto en frecuencia como en duración. Dichas conductas incluyen la preocupación por engordar, los atracones con sensación de falta de control al comer y las conductas alimentarias restrictivas (práctica de dietas, ayunos, ejercicio excesivo y consumo de pastillas para adelgazar) y purgativas (uso de laxantes, diuréticos y vómito autoprovocado) que tienen como fin la reducción de peso ${ }^{8}$.

De acuerdo con la Encuesta Nacional de Salud y Nutrición (ENSANUT), las conductas alimentarias de riesgo más frecuentes en los/las adolescentes mexicanos/as fueron la preocupación por engordar, comer demasiado y perder el control sobre lo que se come. La cantidad de adolescentes que participó en dicha encuesta y que estaban en riesgo de tener un TCA fue del $1.3 \%$ ( $1.9 \%$ en el sexo femenino y $0.8 \%$ en el masculino). Esta proporción fue medio punto porcentual más alta que la registrada en la ENSANUT $2006^{\circ}$, y se registra un incremento nacional entre 2006 y 2012 en la prevalencia de las conductas de riesgo para AN, BN y TCA ${ }^{10}$.

Del mismo modo, en el año 2010, Unikel, et al. ${ }^{8}$ realizaron un estudio en el Distrito Federal con base en la Encuesta Mexicana de Salud Mental Adolescente, en una muestra representativa de 3005 adolescentes de entre 12 y 19 años, y encontraron un $0.2 \%$ de AN y un $0.9 \%$ de $B N$ en las mujeres ${ }^{8}$.

\section{Relación entre los trastornos de la conducta alimentaria y la alexitimia}

Espina $^{11}$ concluye que la alexitimia aparece en diferentes patologías, especialmente en las psicosomáticas, las toxicomanías y los TCA, ya que las personas 
con TCA somatizan sus afectos y actúan de forma impulsiva ante situaciones estresantes sin tener un control adecuado de la toma de conciencia y la expresión de sus afectos.

Mitchell y Mazzeo ${ }^{12}$ describen que la incapacidad de una persona para identificar y discriminar sus estados emocionales puede representar un vacío emocional que genera un exagerado interés en detalles físicos de su propio cuerpo. De igual forma, Cochrane, et al. ${ }^{13}$ analizaron las características individuales de la alexitimia y concluyeron que en los TCA las personas tienen déficits específicos en la identificación y la comunicación de sus sentimientos.

\section{Escala de alexitimia de Toronto}

Para medir el nivel de alexitimia en las personas de la población de Toronto, Bagby, et al. ${ }^{14}$ crearon la Escala de Alexitimia de Toronto (TAS), la cual es ampliamente utilizada. Dicha escala ha tenido dos modificaciones (TAS-R y TAS-20). La versión original de se conforma de 26 apartados que fueron reducidos a cuatro factores. La versión final de la escala consistió en 20 reactivos y tres factores: a) dificultad para identificar los sentimientos y las diferencias entre sentimientos y sensaciones corporales; b) dificultad para describir sentimientos; y c) pensamiento orientado externamente. Según los puntos de corte determinados empíricamente, las personas que obtengan una calificación de 61 o más en la TAS-20 se identifican como pacientes con alextimia $^{14}$.

La TAS-20 se puntúa mediante una escala tipo Likert de cinco puntos que muestra el grado de conformidad/inconformidad con el enunciado. Por medio de esta escala y otras, diferentes autores han encontrado relación entre la alexitimia y los TCA. A continuación se describen algunos estudios.

Díaz y Balbás ${ }^{15}$ estudiaron las variables relacionadas con la alexitimia en 25 pacientes con TCA y 34 personas de un grupo control, por medio de la TAS-20 y la Escala de Evaluación de los Trastornos de Alimentación. Los resultados reflejaron la existencia de niveles medios de alexitimia significativamente superiores en el grupo de sujetos con TCA que en los sujetos del grupo control.

Por su parte, Espina realizó una investigación que tuvo como objetivo indagar sobre la existencia de alexitimia en los padres que tenían una hija con TCA. Evaluó a los padres de 73 mujeres con TCA y a los padres de 72 mujeres sin TCA, utilizando la TAS, el
Inventario de Depresión de Beck, el Cuestionario de Personalidad de Eysenck y la Escala de Autoevaluación de Ansiedad. El autor describe que los padres de hijas con TCA muestran puntuaciones más altas en el TAS-20 en comparación con los del grupo control. Las puntuaciones altas de los padres se asocian con neurosis, ansiedad y depresión. El autor concluye que la alexitimia debe tenerse en cuenta para ayudar a los padres a expresar emociones y que así puedan ser empáticos, ya que ellos son un modelo muy importante para sus hijos, y si los primeros no saben expresar sus sentimientos, los segundos tampoco lo harán ${ }^{16}$.

Speranza, et al. ${ }^{17}$ realizaron un estudio longitudinal de 3 años en el que investigaron el valor pronóstico de las características de la alexitimia en una muestra de pacientes con TCA. Para ello utilizaron la TAS-20 y el Inventario de Depresión de Beck, y los resultados mostraron que la dificultad en la identificación de sentimientos puede influir de forma negativa en la evolución a largo plazo de los pacientes con TCA.

Por su parte, Behar ${ }^{18}$ determinó la presencia de alexitimia y asertividad en diferentes tipos de TCA. Valoró a 108 mujeres con TCA, a las cuales les aplicó el Test de Actitudes Alimentarias, el Inventario de Desórdenes Alimentarios, la TAS-20 y la Escala de Asertividad de Rathus. El estudio dio como resultado que todos los subgrupos alcanzaron puntajes en rango de alexitimia, especialmente las personas con AN, las cuales también presentaron pensamientos más orientados hacia detalles externos, mientras que las personas con BN mostraron mayor dificultad para discriminar señales emocionales.

\section{Validaciones previas de la Escala de Alexitimia de Toronto}

En Ciudad de México se realizó la validación de la escala TAS-20 con 153 sujetos. La confiabilidad se calculó con el coeficiente alfa de Cronbach, que arrojó un valor de 0.875 . El análisis factorial separa solo los dos últimos factores: dificultad para describir sentimientos a los demás y pensamiento orientado hacia lo concreto ${ }^{19}$.

Por su parte, Unikel y Caballero ${ }^{20}$ validaron la escala TAS-20 en México con pacientes que presentaban TCA. Los autores realizan modificaciones a la versión inglesa y a la validación hecha en población mexica$\mathrm{na}^{21}$, en donde solo toman 10 reactivos, los cuales dividen en dos factores: a) dificultad para identificar emociones y b) dificultad para expresar emociones. 
En Puebla también se realizó la validación de dicho cuestionario con 400 estudiantes universitarios, obteniendo un alfa de Cronbach de 0.77 . En este estudio, los resultados denotan la confiabilidad de los dos primeros factores: dificultad para identificar sentimientos y diferenciarlos de las sensaciones corporales o fisiológicas que acompañan a la activación emocional, y dificultad para describir los sentimientos de los demás; quedando fuera el tercero factor, llamado pensamiento orientado hacia lo concreto ${ }^{22}$. Resultados similares muestra Moral ${ }^{23}$, quien estudió las propiedades psicométricas de la TAS-20 y su relación con la deseabilidad social (medida por la escala $L$ del Inventario Multifásico de Personalidad de Minnesota, MMPI) en una muestra de 381 estudiantes en Nuevo León.

Por lo anterior, el objetivo del presente estudio es realizar la validación de la TAS por medio de la consistencia interna y la validez del constructo del instrumento realizando el método de análisis factorial exploratorio, en una muestra proveniente de una clínica especializada en TCA.

\section{Método}

\section{Tipo de estudio}

Estudio de campo, transversal y de carácter psicométrico con diseño de una sola muestra. Se utilizó una muestra de 435 personas con diagnóstico de TCA de acuerdo con los criterios DSM-IV y DSM-IV TR (APA, 1994, 2002), ya que eran los que se encontraban vigentes en el momento de la recolección de los datos. De estas personas, el $16.5 \%$ presentaba AN, el $19.3 \%$ BN, el $6.7 \%$ trastorno por atracón y el 57.5\% TCA no especificado. La media de edad fue de 22 años (desviación estándar: 10.22) (Tabla 1).

\section{Instrumento}

La TAS- $20^{14}$ consta de 20 reactivos con seis opciones de respuesta tipo Likert, que van desde «en total desacuerdo" hasta "totalmente de acuerdo" y se puntúan entre 0 y 5 . Las preguntas 4, 5, 10, 18 y 19 puntúan de forma inversa.

Cabe señalar que en la escala original de 26 ítems ${ }^{24}$ se ofrecían cinco posibles respuestas, que se puntuaban de 1 a 5 , por lo que el máximo posible, utilizando un método $u$ otro, es el mismo (100 puntos), aunque el rango es diferente: 20-100 y 0-100, respectivamente.

La escala se subdivide en tres áreas: a) dificultad para la discriminación de señales emocionales (reactivos 1, 3, 6, 7, 9, 13 y 14); b) dificultad en la expresión verbal de las emociones (reactivos 2, 4, 11, 12 y 17); y c) pensamiento orientado hacia detalles externos (reactivos $5,8,10,15,16,18,19$ y 20).

La versión utilizada en este trabajo es la traducción española y validada ${ }^{25}$.

\section{Procedimiento}

La escala de alexitimia se aplicó a todas las pacientes de la Clínica Ángeles de TCA en un periodo de 9 años. Se aplicó en conjunto con una serie de pruebas psicológicas en una misma sesión; sin embargo, para este estudio se utilizaron únicamente los datos arrojados por la TAS-20 en la versión estandarizada y validada con población española.

\section{Análisis estadístico}

Los reactivos de la escala fueron sometidos a un análisis de consistencia interna mediante un análisis

Tabla 1. Características de la muestra

\begin{tabular}{|c|c|c|}
\hline & $\mathbf{N}$ & $\%$ \\
\hline \multicolumn{3}{|l|}{ Sexo } \\
\hline Femenino & 396 & 91.0 \\
\hline Masculino & 39 & 9.0 \\
\hline \multicolumn{3}{|l|}{ Estado Civil } \\
\hline Soltero & 338 & 77.7 \\
\hline Casado & 49 & 11.3 \\
\hline Divorciado & 17 & 3.9 \\
\hline Viudo & 4 & 0.9 \\
\hline Unión libre & 4 & 0.9 \\
\hline Otro & 23 & 5.3 \\
\hline \multicolumn{3}{|l|}{ Escolaridad } \\
\hline Primaria & 8 & 1.8 \\
\hline Secundaria & 85 & 19.5 \\
\hline Preparatoria & 133 & 30.6 \\
\hline Licenciatura & 188 & 43.2 \\
\hline Posgrado & 6 & 1.4 \\
\hline Otro & 15 & 3.4 \\
\hline \multicolumn{3}{|l|}{ Ocupación } \\
\hline Estudiante & 313 & 72.0 \\
\hline Profesionista & 89 & 20.5 \\
\hline Hogar & 20 & 4.6 \\
\hline Desempleado & 8 & 1.8 \\
\hline Otro & 5 & 1.1 \\
\hline \multicolumn{3}{|l|}{ Tiempo de Evolución } \\
\hline $0-6$ meses & 86 & 19.8 \\
\hline 6-12 meses & 49 & 11.3 \\
\hline $1-3$ años & 90 & 20.7 \\
\hline 3-5 años & 30 & 6.9 \\
\hline 5 años en adelante & 160 & 36.8 \\
\hline Otro & 20 & 4.5 \\
\hline
\end{tabular}


Tabla 2. Reactivos de cada componente con sus respectivas cargas factoriales

\begin{tabular}{llc}
\hline Factor $\mathbf{1}$ & Reactivo & Carga factorial \\
\hline 13 & No sé lo que sucede dentro de mí & 0.83 \\
9 & Tengo sentimientos que no identifico completamente & 0.80 \\
1 & A menudo estoy confuso sobre las emociones que estoy sintiendo & 0.76 \\
6 & Cuando estoy alterado no sé si estoy triste, asustado o enojado & 0.74 \\
2 & Es difícil para mí encontrar las palabras exactas para describir lo que siento & 0.74 \\
7 & A menudo estoy confuso sobre mis sensaciones corporales & 0.73 \\
14 & A menudo no sé por qué estoy enfadado & 0.72 \\
3 & Tengo sensaciones físicas que ni siquiera los médicos comprenden & 0.58 \\
8 & Prefiero dejar que las cosas pasen que entender por qué suceden de esa manera & 0.40 \\
\hline Factor $\mathbf{2}$ & Reactivo & Carga factorial \\
\hline 12 & La gente me pide que exprese mis sentimientos & 0.74 \\
17 & Es difícil para revelar mis sentimientos más íntimos incluso a mis amigos más cercanos & 0.73 \\
4 & Soy capaz de describir fácilmente mis sentimientos & 0.67 \\
\hline 15 & Me es difícil decir qué es lo que siento acerca de las personas & 0.62
\end{tabular}

de confiabilidad alfa de Cronbach, en el programa estadístico SPSS en su version 21. De igual forma, se llevó a cabo un análisis factorial de componentes principales con rotación oblimin.

\section{Resultados}

Se consideraron válidos aquellos reactivos que obtuvieron una correlación ítem-total $\geq 0.28$, por lo cual se eliminaron los reactivos $5,10,16,18,19$ y 20 . Se observó que la mayoría de dichos reactivos correspondían a la subescala «pensamiento orientado hacia lo externo».

Ahora bien, para conocer la estructura psicométrica de la escala, se llevó a cabo un análisis factorial de componentes principales con rotación oblimin con los reactivos restantes, el cual arrojó dos factores después de seis iteraciones que explicaron el $52.58 \%$ de la varianza total.

De esta manera, el instrumento quedó conformado por 14 reactivos divididos en dos factores con nueve y cinco preguntas, respectivamente.

El factor «dificultad para la discriminación de señales emocionales», con una varianza explicada del $43.37 \%$, y el factor «dificultad en la expresión verbal de las emociones», con una varianza explicada del
9.20\%, obtuvieron un alfa de Cronbach de 0.89. Los reactivos de cada componente y sus cargas factoriales se muestran en la tabla 2.

Por último, la escala mostró una consistencia interna aceptable (alfa de Cronbach: 0.89), así como cada uno de los factores $(\mathrm{F} 1=0.87$ y $\mathrm{F} 2=0.75)$.

\section{Discusión}

De acuerdo con el análisis estadístico de validez de la TAS, se eliminaron seis reactivos debido a los criterios estadísticos preestablecidos, por lo que la escala terminó con 14 reactivos, los cuales conforman los primeros dos factores originales, con nueve reactivos para el primer factor («dificultad para la discriminación de señales emocionales») y cinco para el segundo factor («dificultad en la expresión verbal de las emociones»). La varianza explicada del primer factor es del $43.37 \%$, y la del segundo factor es del $9.20 \%$, mientras que la consistencia interna del instrumento es aceptable, ya que cuenta con un alfa de Cronbach de 0.89. Por ello, se considera adecuado haber eliminado el tercer factor. Dichos resultados concuerdan con la mayoría de los estudios revisados ${ }^{20,23}$. 
Según el análisis reportado, se concluye que la escala satisface los criterios de validez necesarios para ser utilizada en dicha población. Ahora bien, fue necesario realizar la validación de la TAS en población con TCA debido a la relación que existe entre estos y la alexitimia. De hecho, Lavender y Anderson ${ }^{26}$ proponen una explicación de dicha relación, la cual define que las conductas de los TCA se desarrollan porque se busca regular las emociones, y las personas con un TCA tienen dificultades al acceso de estrategias efectivas de regulación emocional.

Ahora bien, es esencial incluir intervenciones terapéuticas para las personas en riesgo de desarrollar un TCA, y tal como lo explican Calvo, et al. ${ }^{27}$, en dichos programas de prevención se debe facilitar el reconocimiento, la aceptación y la regulación emocional. De igual forma, es imperativo identificar la alexitimia, que puede asociarse principalmente a la depresión, la ansiedad social y la falta de asertividad en esta población ${ }^{28}$.

\section{Agradecimientos}

\section{A Ana Imelda Hjort Colunga y Elizabeth Arriaga Mendoza.}

\section{Responsabilidades éticas}

Protección de personas y animales. Los autores declaran que para esta investigación no se han realizado experimentos en seres humanos ni en animales.

Confidencialidad de los datos. Los autores declaran que han seguido los protocolos de su centro de trabajo sobre la publicación de datos de pacientes.

Derecho a la privacidad y consentimiento informado. La investigación cumplió con las normas éticas en donde los participantes, en cada Estado respectivamente, firmaron una hoja de consentimiento informado aceptando su participación; asimismo, ninguno de los participantes corrió ningún tipo de riesgo al responder los cuestionarios.

\section{Financiamiento}

El presente estudio no recibió financiamiento alguno para su realización. Cada uno de los autores dedicó su trabajo sin recibir subsidio alguno.

\section{Conflicto de intereses}

No existe conflicto de intereses en ninguno de los autores.

\section{Bibliografía}

1. Beales DL, Dolton R. Eating disordered patients: personality, alexithymia, and implications for primary care. Br J Gen Pract. 2000;50:21-6.

2. Lusiardo M, Marina R. Alexitimia como constructor de la personalidad: concepto, clínica, etiología y enfoque terapéutico. Salud Militar. 2011; 30:48-52.

3. Taylor GJ. The alexithymia construct: conceptualizacion, validation, and relationship with basic dimensions of personality. New Trends in Experimental and Clinical Psychiatriatry. 1996;10:61-74.

4. Gómez JA, Gaite L, Gómez E, Carral L, Herrero S, Vázquez- Barquero JL. Guía de prevención de los trastornos de la conducta alimentaria y sobrepeso. 2008. (Consultado el 26 de noviembre de 2015.) Disponible en: http://saludcantabria.es/uploads/pdf/ciudadania/Guia\%20Prevencion\%20 Trastornos\%20Conducta\%20Alimentaria-2012.pdf

5. Vargas Baldares MJ. Trastornos de la conducta alimentaria. Revista Médica de Costa Rica y Centroamérica. 2013;LXX:475-82.

6. López C, Treasure J. Trastornos de la conducta alimentaria en adolescentes: descripción y manejo. Revista Medica. 2011;22:85-97.

7. American Psychiatric Association. Diagnostic and statistical manual of mental disorders (IV-TR ed.). Washington, DC: APA; 2002.

8. Unikel-Santoncini C, Nuño-Gutiérrez B, Celis de la Rosa A Saucedo de Jesus T, Trujillo Chi Vacuán EM, García-Castro F, et al. Conductas alimentarias de riesgo: prevalencia en estudiantes mexicanas de 15 a 19 años. Revista de Investigación Clinica. 2010; 62:424-32.

9. Barriguete-Meléndez JA, Unikel-Santoncini C, Aguilar-Salinas C, Córdoba-Villalobos JA, Barquera S, Shamah T, et al. Prevalence of abnormal eating behaviors in adolescents: results of a population-based nationwide survey (Mexican Health and Nutrition Survey 2006). Salud Publica Mex. 2009;51(Supl 4):S638-44.

10. Barriguete-Meléndez JA, Campos-Nonato I, Hernández-Barrera L, Barquera S, Córdoba-Villalobos JA, Hernández-Ávila M. Prevalencia de conductas alimentarias de riesgo en adolescentes mexicanos: resultados de la Encuesta Nacional de Nutrición y Salud 2012. Revista Salud Pública de México. 2018; en prensa.

11. Espina A. El constructo alexitimia. Implicaciones clínicas y terapéuticas. En: García J, Garrido M, Rodríguez L, editores. Personalidad, procesos cognitivos y psicoterapia. Un enfoque constructivista. Madrid: Fundamentos; 1988.

12. Mitchell KS, Mazzeo SE. Mediators of the association between abuse and disordered eating in undergraduate men. Eating Behaviors. 2005; 6:318-27.

13. Cochrane CE, Timothy DB, Wilson DB, Hodges EL. Alexithymia in the eating disorders. Int J Eat Disord. 1993;14:219-22.

14. Bagby RM, Parker JDA, Taylor GJ. The twenty-item Toronto Alexithimia Scale-I. Item selection and cross-validation of the factor structure. J Psychosom Res. 1994;38:23-32.

15. Diaz J, Balbás A. Estudio de variables relacionadas con la alexitimia en pacientes con trastornos del comportamiento alimentario. $3 .^{\text {er }}$ Congreso Virtual de Psiquiatría; 2002. p. 1-16.

16. Espina A. Alexithymia in parents of daughters with eating disorders Its relationships with psychopathological and personality variables. J Psycho Res. 2003;55:553-60.

17. Speranza M, Loas G, Wallier J, Corcos M. Predictive value of alexithymia in patients with eating disorders: a 3-year prospective study. J Psychosom Res. 2007;63:365-71.

18. Behar R. Expresión emocional en los trastornos de la conducta alimentaria: alexitimia y asertividad. Revista Chilena de Neuropsiquiatría. 2011:49:338-46.

19. Peréz-Rincón H, Cortés J, Ortiz S, Pena J, Ruiz J, Díaz-Martínez A (1997). Validadación y estandarización de la escala modificada de alextimia de Toronto. Salud mental. 1997;20:30-4. Dispoible en: http://www. inprf-cd.gob.mx/pdf/sm2003/sm200330.pdf

20. Unikel S, Caballero A. La Escala Modificada de Alexitimia de Toronto, validación en pacientes mexicanas con trastorno de la conducta alimentaria. Psicología Iberoamericana. 2005;13(2):88-95. 
21. Pérez-Rincón H, Cortés J, Ortiz-León S, Peña J, Ruiz J, Díaz-Martínez A. Validación y estandarización de la versión española de la Escala Modificada de Alexitimia de Toronto. Salud mental. 1997;20(3):30-4.

22. Weisel M. Validación de la Escala de Alexitimia de Toronto (TAS-20). Tesis. Puebla: Universidad de las Américas; 2007. Disponible en: http://catarina. udlap.mx/u_dl_a/tales/documentos/ps/weisel_d_m/capitulo_4.html

23. Moral J. Propiedades psicométricas de la escala de Alexitimia de Toronto de 20 reactivos en México. Revista Electrónica de Psicología Iztacala. 2008;11(2). Disponible en: http://www.journals.unam.mx/index.php/repi/ article/view/18555.

24. Taylor G, Ryan D, Bagby M. Toward the development a new self-report alexithimia scale. Psychoterapy Psychosomatics. 1985:44:191-9.
25. Moral de la Rubia J, Retamales R. Estudio de validación de la escala de alexitimia de Toronto (TAS-20) en muestra española. Psicologia.com. $2000 ; 4(2): 1-10$

26. Lavender JM, Anderson DA. Contribution of emotion regulation difficulties to disordered eating and body dissatisfaction in college men. Int J Eat Disord. 2010;43:352-7.

27. Calvo R, Solórzano G, Morales M, Kassem MS, Codesal R, Blanco A, et al. Procesamiento emocional en pacientes TCA adultas vs. adolescentes. Reconocimiento y regulación emocional. Clínica y Salud. 2014;25:19-37.

28. Behar R. Funcionamiento psicosocial en los trastornos de la conducta alimentaria: ansiedad social, alexitimia y falta de asertividad. Revista Mexicana de Trastornos Alimentarios. 2010;1:90-101. 